\title{
Mechanic and Acoustic Properties of the Sound-Absorbing Material Made from Natural Fiber and Polyester
}

\author{
Limin Peng, ${ }^{1}$ Boqi Song, ${ }^{1}$ Junfeng Wang, ${ }^{2}$ and Dong Wang ${ }^{1}$ \\ ${ }^{1}$ Research Institute of Wood Industry, Chinese Academy of Forestry, Beijing 100091, China \\ ${ }^{2}$ GuangXi Zhuang Autonomous Region Forestry Research Institute, Nanning 530002, China \\ Correspondence should be addressed to Limin Peng; penglm@caf.ac.cn
}

Received 16 October 2014; Accepted 31 December 2014

Academic Editor: Xinfeng Xie

Copyright (c) 2015 Limin Peng et al. This is an open access article distributed under the Creative Commons Attribution License, which permits unrestricted use, distribution, and reproduction in any medium, provided the original work is properly cited.

\begin{abstract}
A sound-absorbing composite material made of wood fiber and polyester fiber was produced using polyester foam technology and wood-based composite technology. This study investigated the physical and mechanical properties and the effects of the airflow resistivity of the materials and the depth of the cavities behind on sound-absorbing performance of the composite material. The results showed that the composite of best physical and mechanical properties and sound absorption was made with a $150^{\circ} \mathrm{C}$ hotpressing temperature, 10 min hot-press time, $3: 1$ the ratio of wood fiber to polyester fiber, foaming agent content of $8 \%$, and the nominal density of $0.2 \mathrm{~g} / \mathrm{cm}^{3}$; the composite material yielded superior sound absorption property with the airflow resistivity of $1.98 \times 10^{5} \mathrm{~Pa} \cdot \mathrm{s} / \mathrm{m}^{2}$; the acoustic absorption peak moved to lower frequencies when length of the cavities was increased.
\end{abstract}

\section{Introduction}

Noise has become one of the four major pollution types in the world. Constant exposure to noises can cause all kinds of health problems, such as hearing loss, cardiovascular disease, and sleep disorder [1]. It is imperative to produce cost-effective and environmental-friendly materials that can reduce noise pollution.

Natural fibers have been extensively used to produce environmentally friendly composite materials. Natural fibers, such as wood, hemp, and coconut shells, hold great potential for substituting the expensive synthetic fibers in manufacturing acoustic absorption boards due to their abundance, low cost to process, and the natural cellular structure, which can effectively absorb acoustic energy.

Porous materials are important sound-absorbing materials. Studies on the sound absorption properties of various porous materials have been done since 1970s. Delany and Bazley's study on glass fiber and mineral wool found that the absorption coefficient of cellular sound-absorbing materials was lower in low frequency, and it kept an upward tendency as the frequency went up. They established an empirical model of acoustic absorption coefficient with airflow resistivity [2].
However, this method was valid only for limited types of materials with specific characteristics. Luo and Li proposed that natural fiber reinforced composites had better acoustic properties than their synthetic counterparts but still failed to meet the requirements for acoustic materials [3]. The study by Ersoy and Küçük indicated that the absorption peak moved from $6300 \mathrm{~Hz}$ to $4000 \mathrm{~Hz}$ when the thickness of the teafiber materials was doubled [4]. Zulkifli et al. worked on coconut fiber and found that the absorption coefficient could reach 0.70 to 0.80 in the frequency range of $1000-1800 \mathrm{~Hz}$ [5]. Narang studied the influence of the surface density, fiber morphology, and bonding fiber content on the sound absorption property of polyester fiberboard and found that the absorption coefficient increases with increasing surface density at low and medium frequencies. The best absorption property was obtained when the bonding fiber content was $35 \%$ [6]. Küçük and Korkmaz's study on the mix of natural fiber and nonwoven materials showed that the physical properties of the blending materials had a great influence on the sound absorption property of the mixed material. The results showed that as the thickness of the materials increased, the air permeability decreased and the flow resistance was improved, leading to sound absorption enhancement [7]. 
TABLE 1: The physical and chemical properties of the composite materials.

\begin{tabular}{lcccccc}
\hline Text item & Density $\left(\mathrm{g} / \mathrm{cm}^{3}\right)$ & $\mathrm{MC}(\%)$ & $\begin{array}{c}2 \mathrm{~h} \text { thickness expansion } \\
\text { rate of water absorbing }(\%)\end{array}$ & MOR (MPa) & IB (MPa) & $\begin{array}{c}\text { Formaldehyde emission } \\
\text { content }(\mathrm{mg} / 100 \mathrm{~g})\end{array}$ \\
\hline Standard (functions) & $\rho<0.35$ & $4-12$ & $\leqslant 12$ & $\geqslant 2.0$ & - & 9 \\
Text value & 0.20 & 3.7 & 5.9 & 2.6 & 0.42 & 0 \\
\hline
\end{tabular}

Sound absorption mechanisms of the composite were the following three aspects: (1) when acoustic waves propagated into the fibrous material, the air in the fiber pores vibrated and rubbed against cell walls. The generated viscous resistance turned the acoustic energy into thermal energy attenuation. (2) The air in the pores was heated when compressed and it cooled when expanded. The thermal conduction in the materials made acoustic energy transform into thermal energy gradually and it was irreversible. (3) The vibration of fiber itself could also cause the dissipation of acoustic energy. These three aspects cooperated and worked together on the acoustic waves so that the acoustic energy was transferred [8-10].

Polyester fiber is widely used and the polyester fiber board shows the characteristics of good sound absorption property, while the chemically synthesized polyester fiber has the problem of high environmental pollution and high cost. Under the premise in sound absorption, wood fiber was used to substitute parts of the polyester fiber, which could reduce the pollution and lower the cost. In this research, a series of tests were conducted to determine the optimum process parameters for wood fiber/polyester fiber composite sound-absorbing material. The physical and mechanical properties of the material were measured based on the relevant standards. The porous microstructure of the materials was observed with scanning electron microscopy to analyze the mechanism of sound absorption. The sound absorption coefficients under different conditions were tested in transfer function method with impedance tubes. The relationships between the sound absorption properties of composite materials and airflow resistance as well as the thickness of the cavities were obtained after comparison and analysis.

\section{Material and Methods}

2.1. Materials. Poplar wood fiber was obtained from the research forest of Chinese Academy of Forestry. The wood fiber was dried to the moisture content of $2 \%-5 \%$. Polyester fiber was purchased from Shandong Taian plastic factory (PET, solid fiber, with circular cross section and diameter is $30 \mu \mathrm{m}$, length is $6 \mathrm{~mm}$, and specific gravity is 1.36). Under laboratory conditions the wood fiber and polyester fiber were artificially premixed in a ratio of $3: 1$ and then mixed uniformly through wind conveyer. Air-lay web technology was used to get the isotropic structure of the composites. Polyester fiber was fed into the air-laid web-forming machine for opening and mechanical beat and tear to loose fiber bundle and then went through carding machine for combing. Wood fiber was added and the hybrid went through the opener again. Then the mixed fiber was formed under centrifugal force and suction airflow. Isocyanate adhesive
(100\% solid content, tech grade) for binding fibers was purchased from Huntsman Polyurethanes Shanghai, Ltd. The resin content was $12 \%$. The dried hybrid fiber was rolling in the blender while adhesive was sized in the form of spray. Then $8 \%$ foaming agent (modified foamer ADC, purchased from Shanghai Fine Raw Chemicals Co., Ltd., tech grade) was added to improve the porosity. ADC released nitrogen, carbon monoxide, and ammonia when heated. ADC was an exothermic foaming agent which might release uncontrollable heat and decreased the viscosity of melt. This would generate a lot of bubbles and coupled together to form a larger aperture, the material constituting the porous internal structure of the voids increased. The decomposition temperature of pure $\mathrm{ADC}$ was 190 to $210^{\circ} \mathrm{C}$, while it was 135 to $145^{\circ} \mathrm{C}$ for modified $\mathrm{ADC}$, which was consistent with the curing temperature of adhesive.

The preformed fiber was preloaded and then hot pressed for $10 \mathrm{~min}$ under the hot press (produced by Shanghai woodbased panel mechanical factory, Model QD) at $150^{\circ} \mathrm{C}$. The target density of composite fiber board was $0.2 \mathrm{~g} / \mathrm{cm}^{3}$, and the finish size was $400 \mathrm{~mm} \times 400 \mathrm{~mm} \times 10 \mathrm{~mm}$. These panels were then cut into test samples fitting the relevant standard for the following tests.

\subsection{Methods}

2.2.1. Sound Absorption Measurement. According to the relevant testing standard [11], using impedance tubes (model number: UA-1630, Bruel \& Kjar Co., Ltd., Demark), the experiment was conducted in a semianechoic room at $20.0^{\circ} \mathrm{C}$ and $50 \%$ relative humidity $(\mathrm{RH})$. For each variable 1500 separate data points in the frequency range of $50-6400 \mathrm{~Hz}$ were obtained every $4 \mathrm{~Hz}$.

2.2.2. Airflow Resistance Measurement. According to the relevant testing standard [12], the airflow resistivity was measured with the test system of flow resistance instrument adopting water tank method (Figure 1) in direct current (DC) method. The differential pressure levels above and beneath the specimen surface were measured by controlling the transmit time of one-way flow through the cylindrical tube.

\section{Results and Analysis}

3.1. Physical and Mechanical Properties of Composite Material. The properties of the composite material are listed in Table 1. The standard values of the properties required by the Chinese Industry Standard LY/T 1718-2007 light fiberboard [13] are listed as well for comparison. The composite material samples were manufactured using the following parameters: hotpressing temperature, $150^{\circ} \mathrm{C}$; composite time, $10 \mathrm{~min}$; wood 


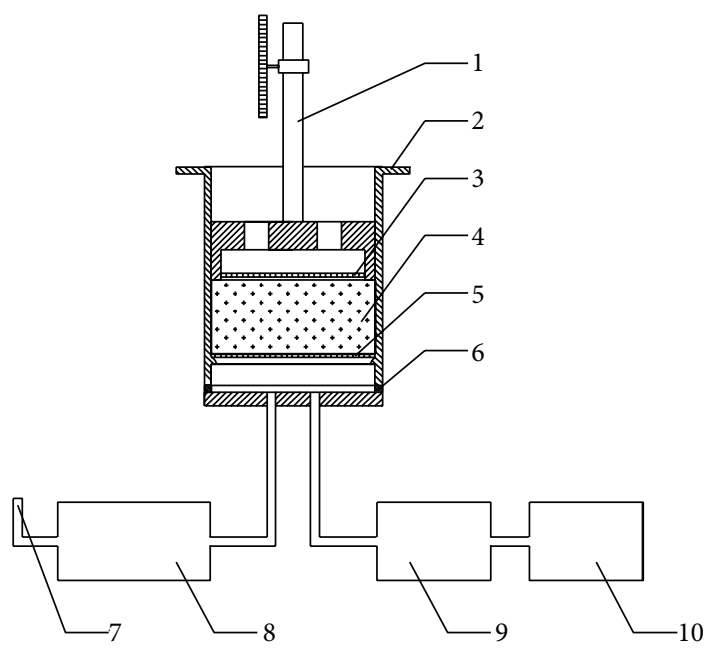

FIGURE 1: Measurement equipment in cross section by DC method. 1: piston with thickness indicator. 2: measure specimen cylinder barrel. 3: grid or perforation plate. 4: specimen. 5: grid or perforation plate for supporting the specimen. 6: seal ring. 7: opening to the atmosphere. 8: differential pressure measuring equipment. 9: flow meter. 10: air supply.

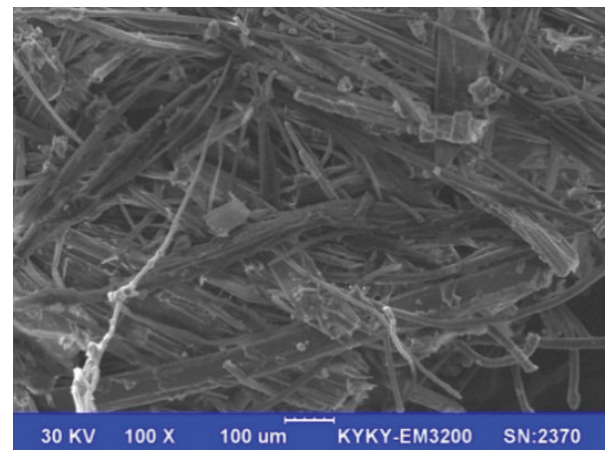

FIGURE 2: SEM of composite material at 100x.

fiber to polyester ratio, $3: 1$; foaming agent content, $8 \%$; and the target density, $0.2 \mathrm{~g} / \mathrm{cm}^{3}$.

As shown in Table 1, the moisture content (MC) of the composite material was lower than the standard requirement. This was due to the low hygroscopicity of polyester fiber $(\mathrm{MC}<0.5 \%)$ and the low moisture content of dried wood fiber $(2-5 \%)$. The 2-hour thickness expansion rate of water absorption and the MOR of the composite material exceeded the standard requirements. The IB of the composite material was $0.42 \mathrm{MPa}$, indicating that the material was strong enough to survive regular handling and installation.

\subsection{Acoustic Properties of the Composite Material. Figures} 2 and 3 show the structures of the material under different magnifications.

As shown in Figure 2, the material contained irregular pore structures composed of interlaced fibers. Figure 3 showed the holes formed from solidified adhesive under the effect of foaming agent. These SEM images showed that

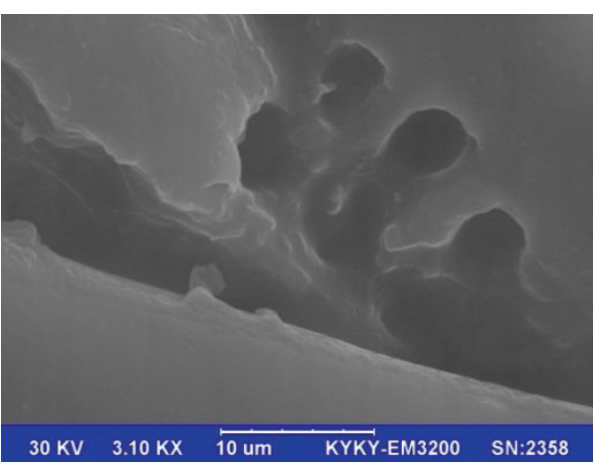

FIgURE 3: SEM of foaming structure of the composite at $3.10 \mathrm{kx}$.

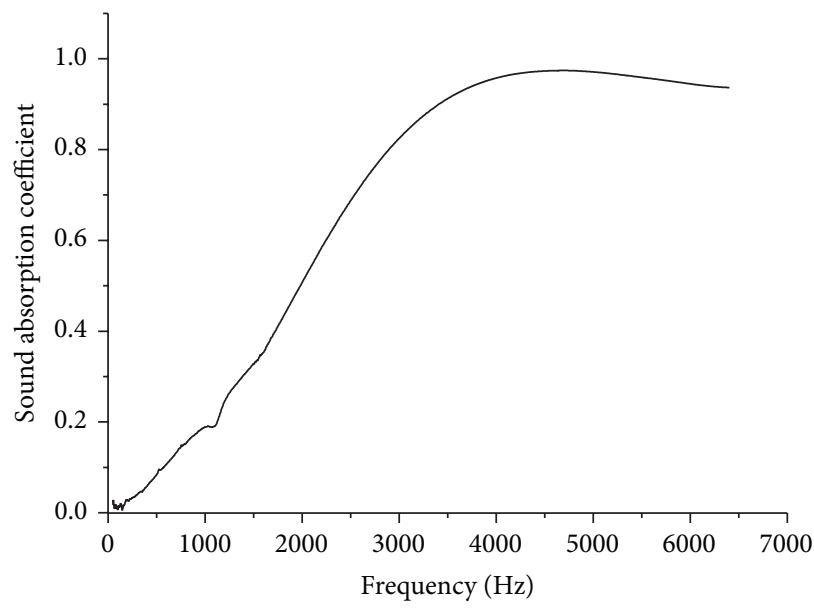

FIGURE 4: The absorption coefficient of wood fiber/polyester fiber composite materials.

the composite pores were made of the voids between the fibers and those formed by the foaming agent.

The sound absorption coefficient of the wood fiber and polyester fiber composite material is shown in Figure 4. The composite material had higher sound absorption coefficients at high frequency range. The absorption coefficient was more than 0.8 in the frequency range of $2892-6500 \mathrm{~Hz}$, and the highest absorption coefficient was 0.97 at $4660 \mathrm{~Hz}$. The wood fiber/polyester fiber composite materials were fibrous porous composite materials where interlaced wood fiber and polyester fiber constituted similar pore structures inside and outside; thus it created the conditions for the entrance to the materials for acoustic waves.

\subsection{Factors Affecting the Acoustic Properties of the Composite Material}

3.3.1. The Influence of Airflow. The air permeability of the materials can be characterized in terms of airflow resistance, which is measured by the ratio of static differential pressure between both sides of the specimen to the airflow velocity when air flows through the materials. Flow resistivity is the flow resistance per unit thickness of the tested material. The flow resistivities of 3 samples with different densities 


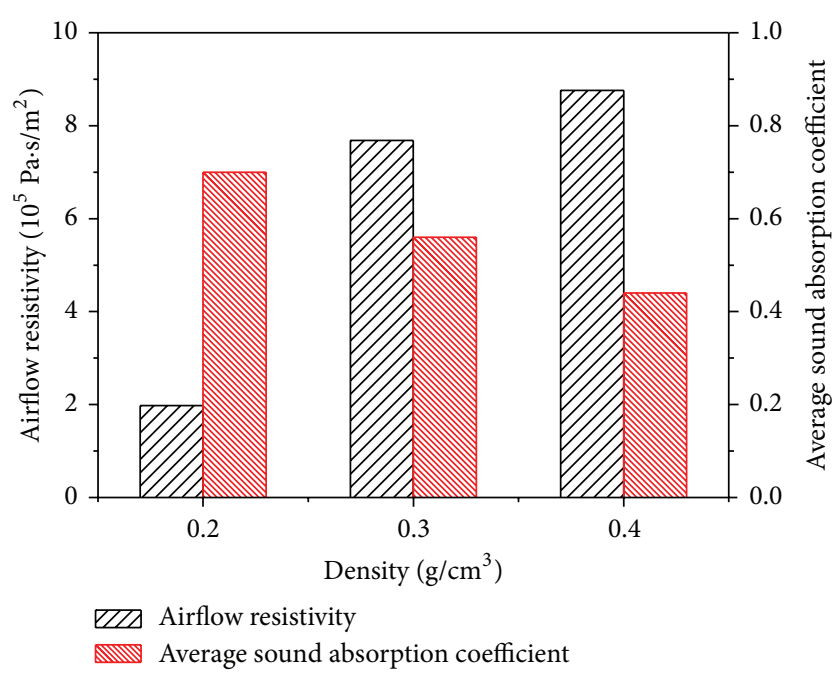

FIGURE 5: The relationship between the average sound absorption coefficient and airflow resistivity of the composites of different densities.

were measured. The average absorption coefficient was the arithmetic mean of 6 sound absorption coefficient values at the central frequencies of 1 octave band width range 125 4000 $\mathrm{Hz}$. Figure 5 showed the relationship between the average absorption coefficient and airflow resistivity of the composite.

The average absorption coefficients of each sample were $0.70,0.56$, and 0.44 , respectively. As shown in Figure 5, the sound absorption coefficient decreased sharply as the flow resistivity went up. And there was a strong correlation between airflow resistivity and density.

Actually, as the density and airflow resistivity increased, the average sound absorption coefficient was firstly increased and then decreased. In a certain range of the thickness of the materials, the larger density means the denser structure. The resistance of air particle through the material was increased, and the average sound absorption coefficient was decreased. There was a best airflow resistivity $\delta_{0}$ and corresponding density $\rho_{0}$ for certain composite fibrous material. When the composite density was less than $\rho_{0}$, there was more and larger voids inside the material and less acoustic wave reflection and refraction. So it was easy for acoustic waves to propagate through the material. Moreover, the internal surface area was small; the friction and viscous resistance between the vibrating air particle and fiber was low. Hence the sound absorption property was poor. In addition, when the density was larger than $\rho_{0}$ and the flow resistivity was too high, most of the acoustic energy was reflected at the surface rather than transmission so that the absorption properties were decreased. According to Hong's research, as the flow resistivity went higher, the absorption coefficient was reduced at almost all frequencies [14]. However, the flow resistivity had only a small effect on the peak location of the absorption coefficient. The above observation of the decrease of absorption coefficient was reasonable as a high flow resistivity tended to reflect the incoming acoustic wave rather than absorbing the sound wave. The sound absorption

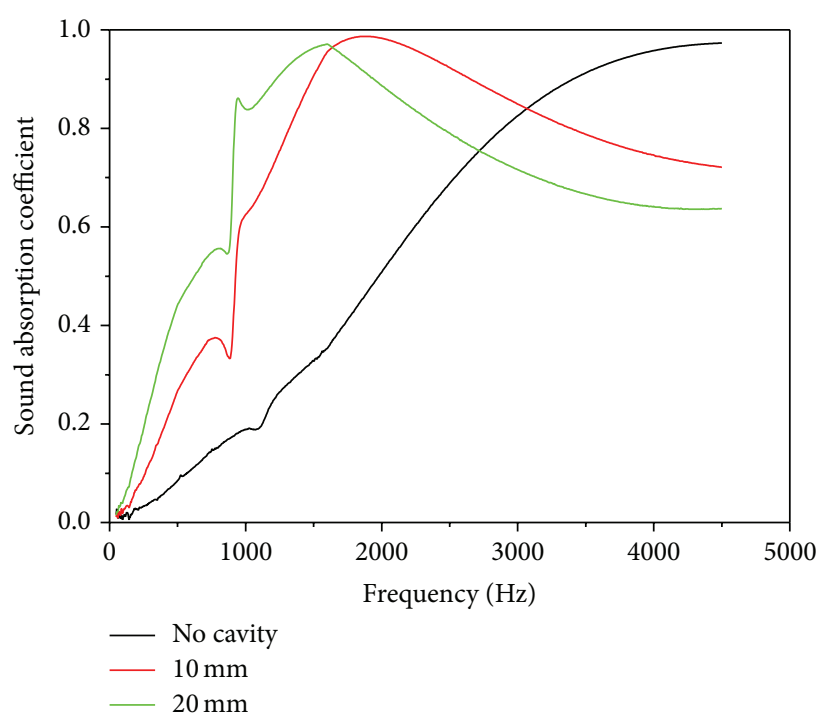

FIgURE 6: The sound absorption coefficient of the composites under different thickness of the cavity.

properties of the materials could be adjusted by controlling the flow resistance. The best airflow resistivity for wood fiber/polyester fiber composite sound-absorbing materials was $1.98 \times 10^{5} \mathrm{~Pa} \cdot \mathrm{s} / \mathrm{m}^{2}$.

3.3.2. The Influence of the Depth of the Cavity. As shown in Figure 6, the absorption coefficient of material with a cavity of $10 \mathrm{~mm}$ thickness between the composites and rigid back was higher than that of materials without cavities behind at the frequency range of 0 to $3000 \mathrm{~Hz}$, and it was increased significantly at the frequency range of 50 to $1500 \mathrm{~Hz}$. When the thickness of the cavity between the composites and rigid back was increased up to $20 \mathrm{~mm}$, the absorption coefficient was higher than that of cavity thickness of $10 \mathrm{~mm}$ at the frequency range of 0 to $1500 \mathrm{~Hz}$, while it decreased quickly at the frequencies from $1500 \mathrm{~Hz}$. Consequently, it could largely enhance the sound absorption property of the composite materials at low and medium frequency ranges by setting cavities of a certain thickness behind them, whereas the enhancement became less obvious when the thickness increased up to a certain value; meanwhile the absorption coefficient was decreased obviously at high frequency. In a practical application, there is usually a certain depth of cavity between material and rigid back to enhance the sound absorption property in low frequency, which can be seen as enlarging the thickness of the materials. In conformity to the acoustic principle, when the thickness of the rear air layer equaled odd-numbered quarter wavelength, the sound absorption coefficient increased to the maximum. It is because the sound pressure $1 / 4$ far from rigid backing was zero and the air particle vibration velocity was maximum so that the sound energy loss caused by friction damping was maximum. In other words, the acoustic energy was utmost absorbed by the composite material, while the sound pressure which was integer times of $1 / 2$ wavelength far from the rigid 
backing was maximum and the particle vibration velocity was zero. Accordingly, the sound absorption coefficient was minimum at the relevant frequency. This character is often used for material savings in construction.

\section{Conclusions}

Results obtained from this study led to the following conclusions.

(1) The composite materials yield superior physical and mechanism properties in the conditions in which the hot-pressing temperature was $150^{\circ} \mathrm{C}$, composite time was $10 \mathrm{~min}$, the ratio of wood fiber to polyester was $3: 1$, the resin content was $12 \%$, foaming agent content was $8 \%$, and the density was $0.2 \mathrm{~g} / \mathrm{cm}^{3}$.

(2) The sound absorption property of the wood fiber/polyester fiber composite was related to the airflow resistivity. The sound absorption coefficient of the material increased as the airflow resistance values decreased. When the airflow resistivity continued to reduce and exceeded the optimum value, the sound absorption coefficient of the materials showed a trend of decrease. The optimum airflow resistivity value of the composite materials was $1.98 \times 10^{5} \mathrm{~Pa} \cdot \mathrm{s} / \mathrm{m}^{2}$.

(3) When there were cavities behind the composite material, the sound-absorbing peak value moved to lower frequencies. As the thickness of the cavities increased, the increase of the sound absorption coefficient in the low frequency range became less obvious.

\section{Conflict of Interests}

The authors declare that there is no conflict of interests regarding the publication of this paper.

\section{Acknowledgments}

The authors would like to thank Chinese Academy of Forestry for the financial and technical support through The State Bureau of Forestry 948 Project Introduction of international advanced forestry science and technology plan "Manufacturing technology recommendation of high performance wood based composite sound absorbing materials" (2013-4-15).

\section{References}

[1] L. L. Shi, Labotary study of the noise annoyance of the low frequency noise [Ph.D. thesis], Zhejiang University, 2004.

[2] M. E. Delany and E. N. Bazley, "Acoustical properties of fibrous absorbent materials," Applied Acoustics, vol. 3, no. 2, pp. 105-116, 1970.

[3] Y. Luo and Y. Li, "Acoustical studies of natural fiber reinforced composites," Journal of Materials Engineering, no. 4, pp. 51-54, 2010.

[4] S. Ersoy and H. Küçük, "Investigation of industrial tea-leaffibre waste material for its sound absorption properties," Applied Acoustics, vol. 70, no. 1, pp. 215-220, 2009.
[5] R. Zulkifli, M. J. Mohd Nor, M. F. Mat Tahir, A. R. Ismail, and M. Z. Nuawi, "Acoustic properties of multi-layer coir fibres sound absorption panel," Journal of Applied Sciences, vol. 8, no. 20, pp. 3709-3714, 2008.

[6] P. P. Narang, "Material parameter selection in polyester fibre insulation for sound transmission and absorption," Applied Acoustics, vol. 45, no. 4, pp. 335-358, 1995.

[7] M. Küçük and Y. Korkmaz, "The effect of physical parameters on sound absorption properties of natural fiber mixed nonwoven composites," Textile Research Journal, vol. 82, no. 20, pp. 2043-2053, 2012.

[8] W. D. Yang and Y. Li, "Sound absorption performance of natural fibers and their composites," Science China Technological Sciences, vol. 55, no. 8, pp. 2278-2283, 2012.

[9] J. F. Allard, Propagation of Sound in Porous Media: Modelling Sound Absorbing Materials, John Wiley \& Sons, London, UK, 2009.

[10] X. Z. Zhong, The Construction Materials for Sound Absorption and Insulation, Chemical Industry Press, Beijing, China, 2012.

[11] ISO, "Acoustics-determination of sound absorption coefficient and impedance in impedance tubes-part 2: transfer function method," ISO 10534-2, International Organization for Standardization, 1998.

[12] ISO 9053: 1991, Acoustics-Materials for Acoustical Applications-Determination of Airflow Resistance, 1991.

[13] LY/T 1718-2007, "Light fiberboard," 2007.

[14] K. W. Hong, Acoustical estimation of macroscopic properties of poroelastic materials [Ph.D. thesis], Purdue University, West Lafayette, Ind, USA, 2009. 

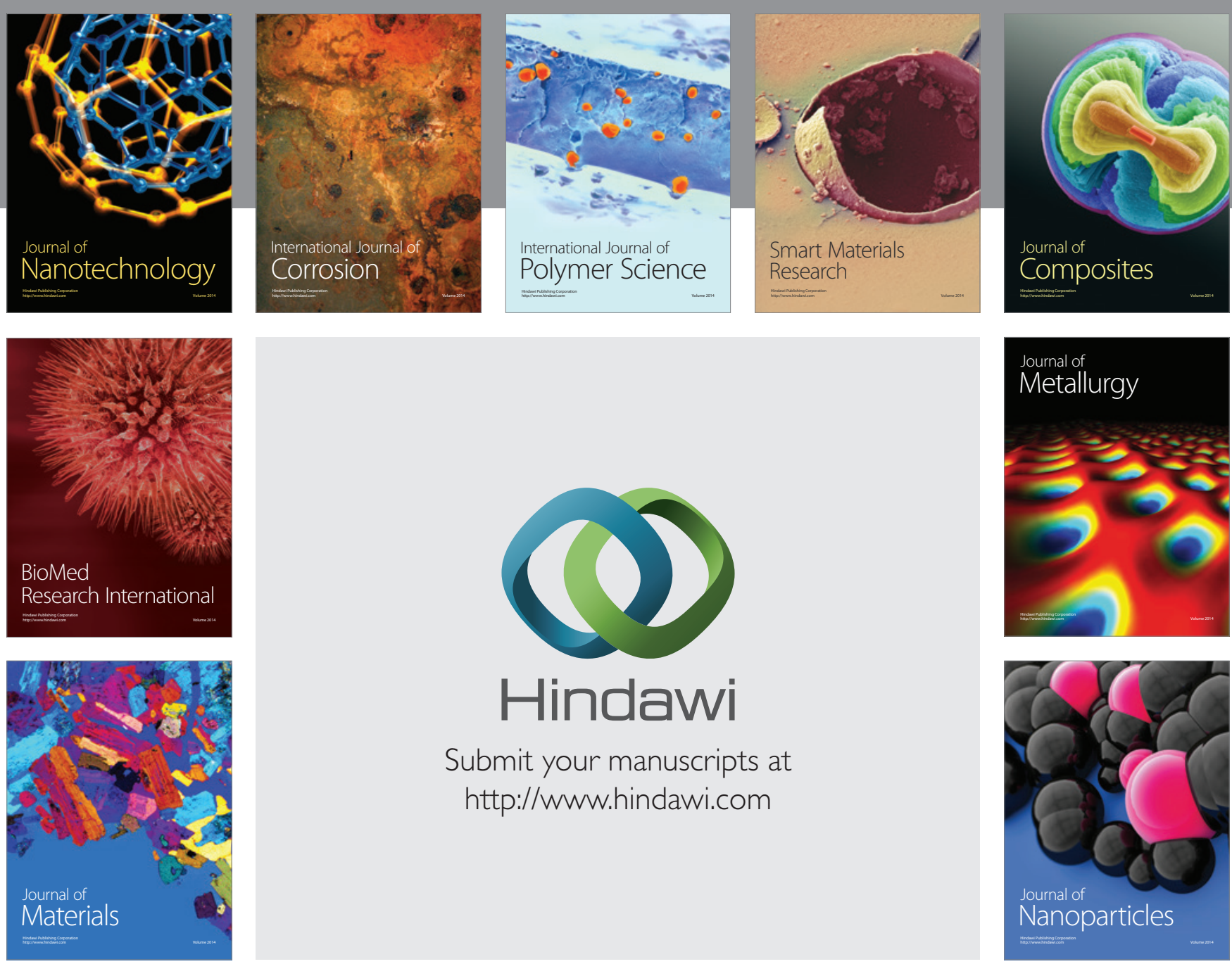

Submit your manuscripts at http://www.hindawi.com
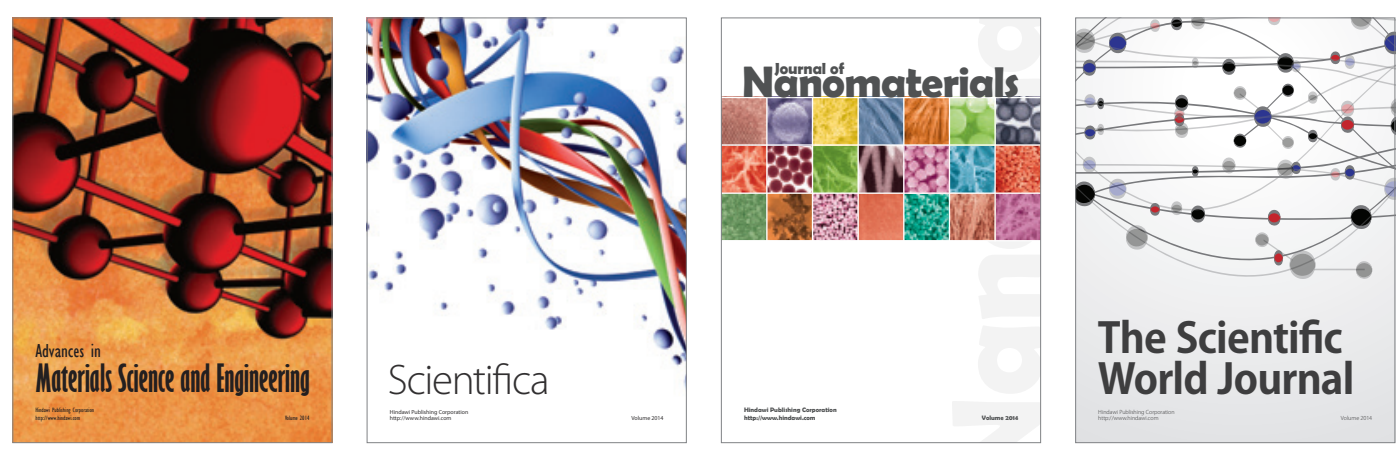

\section{The Scientific World Journal}
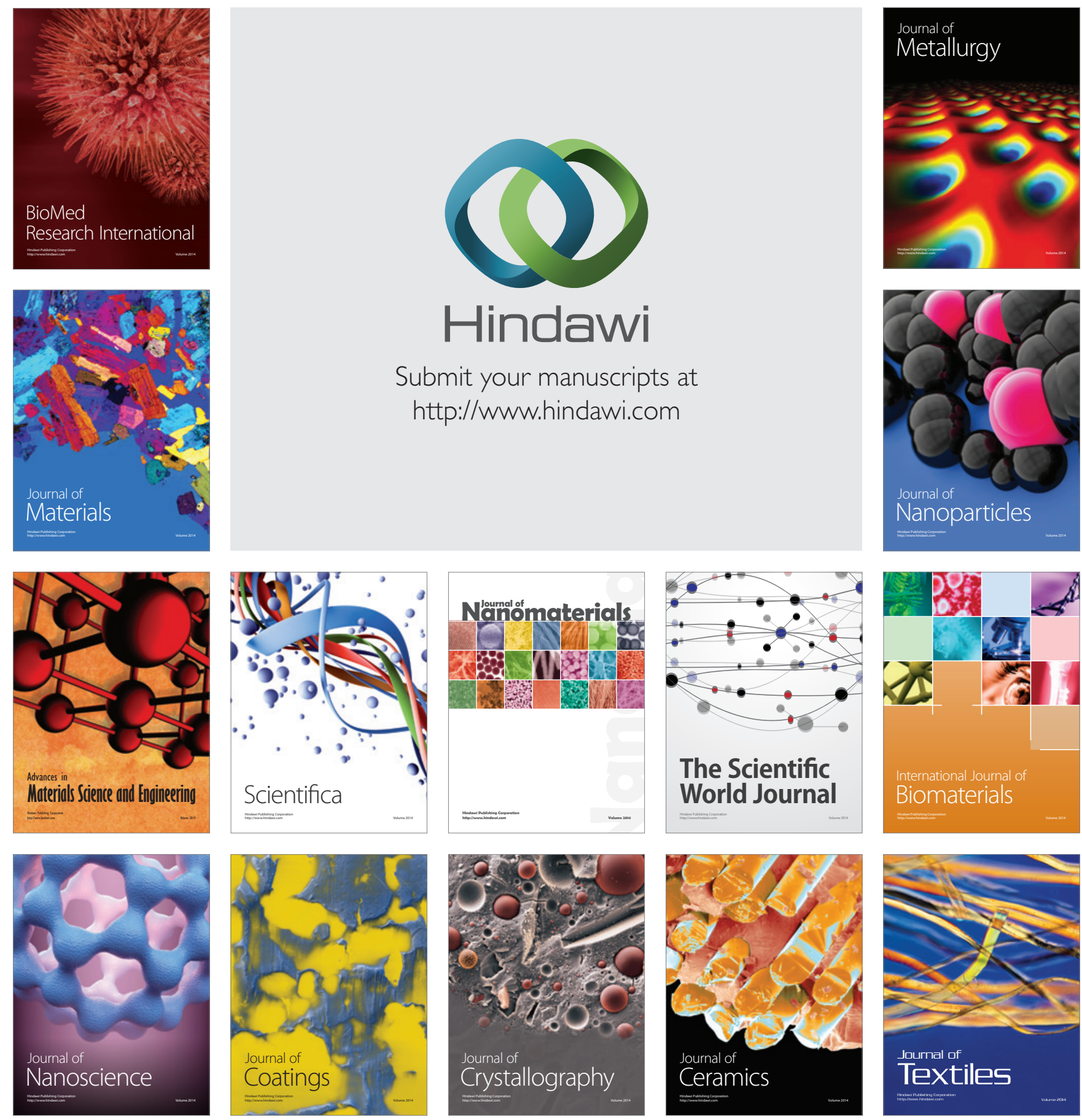